\title{
El Legado del
}

Dr. Richard Kim:

Una Entrevista

con Brian Ricci

Robert Toth

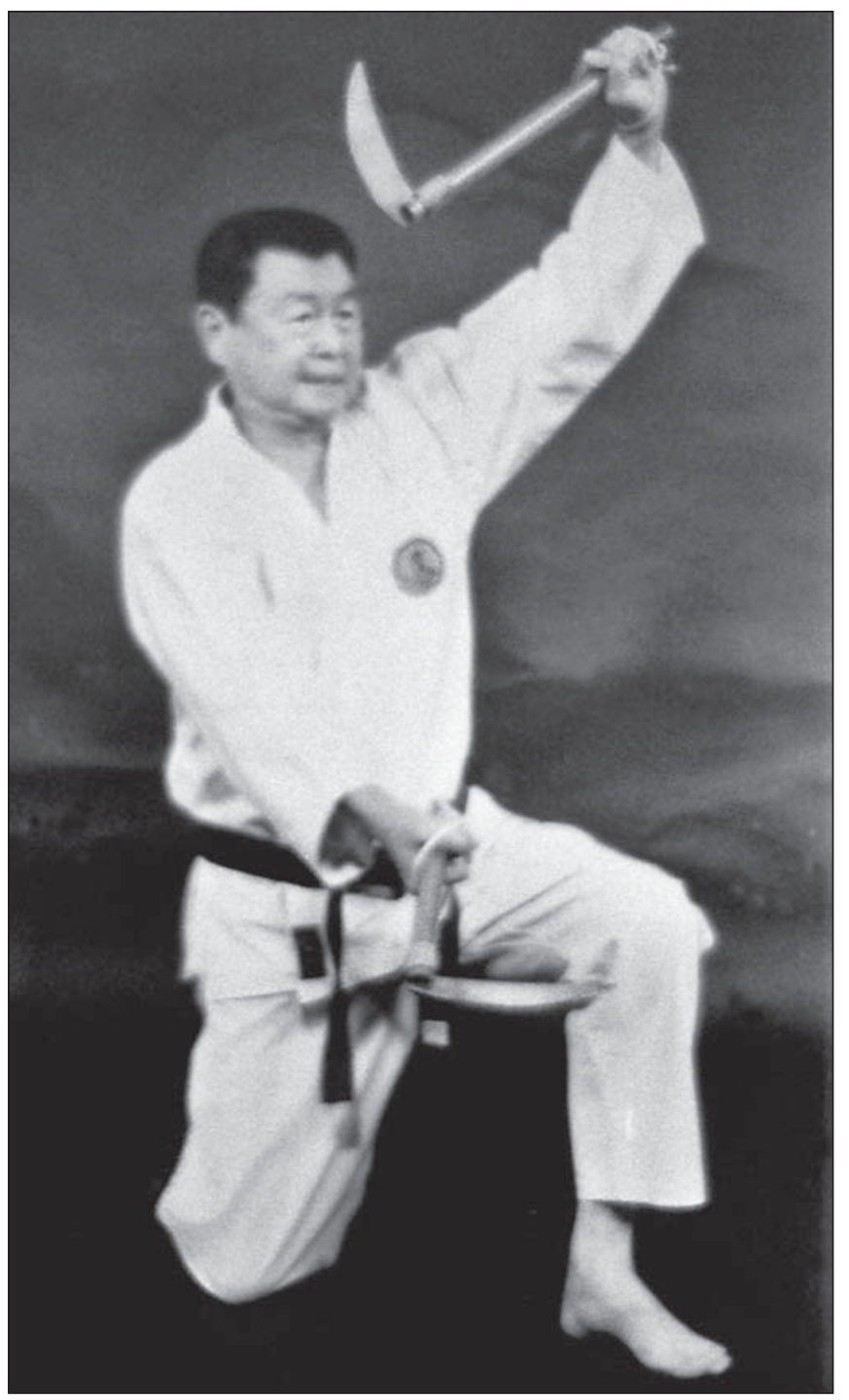

El Dr. Kim en una demostración.

\section{Introducción}

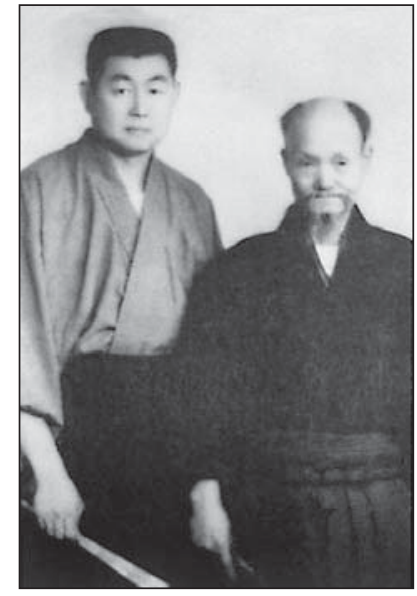

Richard Kim con Yoshida Kotaro.
De la misma manera que un artista usa diversos medios como la arcilla, la pintura o la música como medios de expresión artística, un artista marcial usa su propia vida (Kim, 1988). Richard Kim fue uno de estos artistas. Su obra artística completa, su vida, fue más lejos de lo que muchos podrían llegar a imaginar, y mucho menos conseguir. Si para pagar lo que uno debe a su profesor, el estudiante debe sobrepasarle, entonces Richard Kim puso el listón muy, muy alto. Fue boxeador, militar, sacerdote (Warrener, s.f.), doctor en filosofía oriental (Warrener, 1982: 65), escritor y profesor de universidad, lingüista capaz de hablar seis idiomas (Ricci, 2005, 17 mayo), y profesor de artes marciales (sensei).

Sensei es un término que significa "maestro" o "instructor", y que se usa en todas las artes de Japón y Okinawa. En japonés se escribe con dos símbolos, sen (antes) y sei (vida). Dentro del contexto de las artes marciales, el Sensei es la persona con mucha experiencia en la vida, tanto en la vida en general como en las artes marciales (Castilonia, 1996: 143, 144).

Los sensei se encargan de que sus alumnos desarrollen su potencial hasta el punto más alto y se les considera indicadores del Camino (Do) (Sell 2000: 369). 
Los sensei son responsables de supervisar el entrenamiento y el desarrollo personal de sus estudiantes. Ellos ostentan el respeto y la autoridad absoluta. Los estudiantes, a cambio, aceptan la autoridad de su sensei y siguen sus enseñanzas sin preguntas (Wingate, 1993: 24).

El sensei debe ejemplificar constantemente los más altos estándares de la disciplina de las artes marciales, con la idea de que esta es la forma de desarrollar el carácter. El Camino de las artes marciales debe ser el camino de vida del sensei, entregándose completamente a su arte con el objeto de comunicar de forma completa las enseñanzas de las artes a sus alumnos (McCarthy, 1987: 10). El Doctor Kim fue un sensei ejemplar.

\section{Una breve panorámica de la historia de vida del Dr. Kim}

Richard Kim, de padre coreano y madre japonesa, nació el 17 de noviembre de 1919 en Hawai (Warrener, 1982: 91). Su padre fue arquitecto de parques y jardines y su madre era propietaria de un hotel (Ricci, 2005, 4 mayo). El sótano del hotel se alquilaba a Tachibana, un instructor de judo. Cuando Richard contaba con seis años, su madre lo apuntó a las clases de judo (Warrener, s.f.).

Kim empezó a entrenar karate en 1927 con Arakaki Ankichi (1899- 1927), discípulo de Yabu Kentsu (Kim, 1974: 3). Yabu Kentsu había sido estudiante de dos grandes maestros del kárate okinawanse, Matsumura Sokon y Yasutsune Itosu. Yabu fue uno de los primeros hombres que enseñaron artes marciales en el sistema escolar de Okinawa y se le conocía como "El Sargento" (McCarthy, 1987: 32). En 1927, en su regreso a Okinawa después de una visita a California, Yabu hizo una escala en Hawai. El 8 de julio de 1927 realizó una demostración en la Asociación de Jóvenes Cristianos de Nuuanu (Svinth, 2001: 10, 14). Después de ver la misma, Richard Kim comenzó a entrenar en el estilo Yabu Shorinji-ryu (Warrened, s.f.), el cual es una síntesis del kárate de Okinawa y de Japón (Farkas \& Corcoran, 1983: 242). En la década de los treinta, Kim se encontró nuevamente con Yabu Kentsu en Japón y continuó su entrenamiento con él (Ricci, 2005, 21 abril).

"Biggie" [El importante] Kim, como era conocido, pasó mucho tiempo en clubes de boxeo locales durante la adolescencia, donde adquirió las habilidades del boxeo al actuar de sparring para algunos de los boxeadores de mayor nivel mundial (Kim, 1982:6, 7). Posteriormente explicó que lo que más había aprendido del boxeo fue el jab y a concentrarse. También aprendió las limitaciones del boxeo cuando fue testigo de cómo un boxeador de pesos ligeros perteneciente al Hall of Fame [Vestíbulo de la Fama] daba con su cabeza en el suelo a manos de un samoano (Ricci, 2005, Abril 21). Kim sustuvo 42 combates en el ring y llegó a ser campeón de Oriente mientras vivía en Shanghai (Warrener, 2001: 93).

Después de graduarse en la escuela secundaria, Kim asistió a la universidad de Hawai. Allí se unió al Reserve Officers Training Corp - ROTC [Cuerpo de Entrenamiento de Oficiales Reservistas] y fue ascendido a capitán. A los hombres que completaban el entrenamiento se les licenciaba en la reserva del ejército de los EEUU (www.hawaii.edu/armyrotc).

En 1939 Kim acordó viajar a Japón trabajando como forma de pago (Warrener, s.f.). Tuvo esta posibilidad porque había nacido antes del Acto de Exclusiones de 1924 y tenía la doble nacionalidad (Warrener 2001:93). Pero, a su llegada, tendría que abandonar el barco porque no tenía la edad legal (Ricci, 2005, 21 abril). Una vez en Japón, Kim llegó a ser miembro del ejército japonés (Warrener 2001:92).

En Japón, Kim entrenó con Yoshida Kotaro en la Daito-ryu aikijutsu (Warrener, s.f.). La Dayto-ryu fue uno de los más renombrados estilos de combate japonés antiguo, siendo practicado por el clan de los guerreros Minamoto durante varios siglos antes de que la familia Takeda lo heredara (Ratti \& Westbrook, 1973: 356). Kotaro había entrenado con Takeda Sokato (Warrener, s.f.).

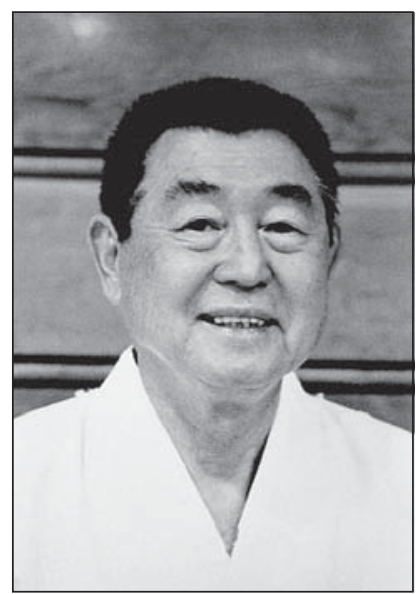

El Dr. Richard Kim. 
Yoshida Kotaro fue famoso por sus habilidades con las armas (Sells, 2000: 135,137 ) y fue experto con la lanza (yari) y con la espada larga (naginata) (Corcoran et al., 1993: 396). El entrenamiento con Yoshida Kotaro era muy físico y muy duro. Kotaro le enseñó técnicas de proyección y también trabajaron con espadas y cuchillos. Kim llegó a ser un aprendiz de Kotaro y con el tiempo le otorgaron el Daito-ryu menkyo kaiden (Warrener, 2001: 92). El menkyo kaiden es un certificado que atestigua la completa capacidad del estudiante y normalmente se otorga al estudiante avanzado juzgado como el más capaz para continuar con el arte (Farkas \& Corcoran, 1983:177).

Richard Kim fue enviado entonces a China como intérprete de la armada imperial japonesa (Ricci, 12 diciembre, 2005). Allí tuvo la oportunidad de estudiar artes marciales. Chen Chin-wan le enseñó una versión ligeramente modificada del estilo Yang de taijiquan. Aprendió baguazhang con Chao Hsu-lie al que conoció mientras estuvo en Hong Kong. Kim también entrenó el gongfu del yiquan con Wang Xiangzhai (1885/6-1963). Su primera lección fue estar en una postura qigong conocida como "abrazar el árbol" durante tres horas al día (Warrener, s.f.). Mientras vivió en Shanghai, Kim también asistió a la Universidad de San John (kim, 1982: $6,7)$.

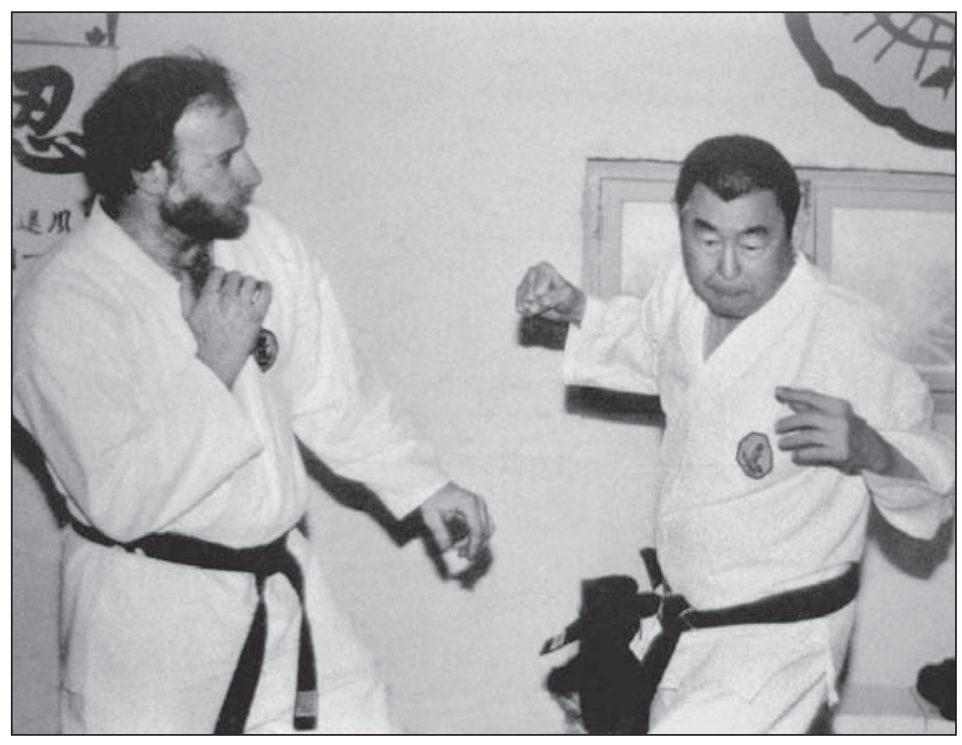

Después de la Segunda Guerra mundial, Kim regentó y dirigió un bar en Yokohama, Japón. Mas Oyama ${ }^{1}$ y Kinjo Hiroshi irían una vez por semana a su casa para entrenar. Más tarde conoció a Yamaguchi Gogen ${ }^{3}$ a través de Oyama (Warrener, s.f.). Durante esta etapa, Kim representó a la Hermandad de Marineros en Yokohama (Kim, 1999).

En 1959, Kim se trasladó a San Francisco, California (Kim, 1974: 3). Dirigió un programa de artes marciales en la Asociación de Jóvenes Cristianos Chinos hasta su semi-jubilación en 1978. Durante todos estos años, el Dr. Kim viajó alrededor de todo el mundo enseñando artes marciales. Creó una gran organización internacional, la Zen Bei Butokukai ${ }^{4}$, con escuelas en los EEUU, Canadá y Europa. Zen Bei se traduce literalmente como "todo el

El Dr. Kim demostrando una técnica de boxeo en Hamilton, Ontario. arroz". Los caracteres japoneses, sin embargo, pueden tener diversos significados, dependiendo del contexto. El carácter para "arroz" puede referirse a "las personas en general". Por eso, Zen Bei puede ser también traducido como "todo el mundo" (Foley, 2005, 3 de Julio).

En el 2000, El Kodanshakai Karate de Hawai concedió al Dr. Kim el cinturón negro décimo grado (Goodin, 2005). En el mismo año, en una reunión llevada a cabo en un restaurante Chino de Sacramento, el Doctor Kim anunció que había otorgado la categoría de séptimo grado a su alumno más antiguo, Brian Ricci. Esta fue la única vez que concedió ese rango a alguien. Fue el dan más alto que concedió (Ricci, 2005, 22 de mayo).

El sensei Kim no asistió al campamento anual de verano de la Zen Bei Butokukai en el 2001, que tuvo lugar en la Universidad Guelph en Ontario, Canadá, debido su débil salud. Dispuso que Brian Ricci dirigiese el campamento en su ausencia. En un encuentro de cinturones negros durante la primera tarde del campamento de verano, Ricci explicó que suponía que algún día el maestro Kim no podría enseñar, pero que no esperaba que esto fuese a pasara tan pronto. Ricci quiso dejar claro que se le había puesto a cargo del campamento e intentó cumplir con su responsabilidad hacia su maestro. 
Richard Kim murió el 8 de noviembre del 2001. Brian Ricci ha continuado con el trabajo de su maestro difundiendo las artes marciales. Ahora la mayor parte de los estudiantes del sensei Kim entrenan con el sensei Ricci. El campamento internacional de verano Zen Bei Butokukai en la universidad de Guelp continua manteniéndose cada año y ha crecido bajo la atención del maestro Ricci con estudiantes provenientes de todas partes de Norteamérica.

\section{Entrevista con Brian Ricci}

La siguiente entrevista con el maestro Brian Ricci fue llevada a cabo por Robert Toth el 21 de abril de 2005 en St. Catharines, Ontario.

\section{RT ¿Cuándo y dónde naciste?}

BR Nací el 14 de agosto de 1950 en Everett, Massachussets, a seis millas del centro de Boston. Hoy en día vivo a media milla de donde nací.

\section{RT ¿Cuándo se produjo su primer contacto con las artes marciales y por qué?}

BR Siempre he sido un aficionado a las películas. Viendo a Jimmy Cagny haciendo judo en Blood on the Sun [Sangre sobre el sol] o en las películas de. Sr. Moto. Nadie sabía que el kárate había regresado, pero yo sí lo sabía y quería aprender cómo

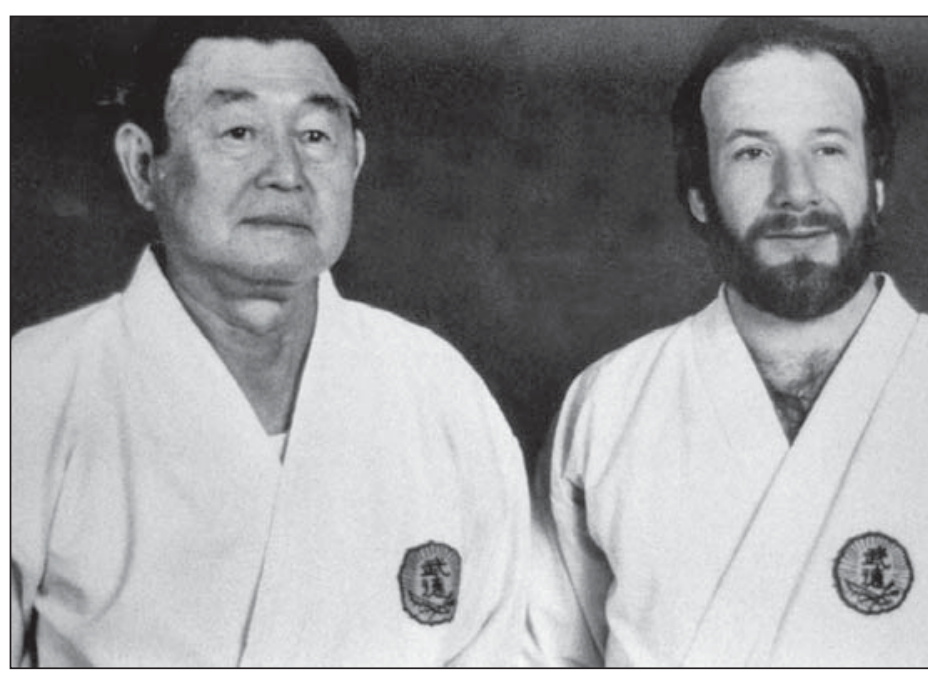
hacer esas técnicas.

Sería sobre 1962 cuando iba con mi madre y le dije que quería aprender jujutsu. Ella me dijo que consiguiera un libro. Cuando tenía unos catorce años, le dije que quería aprender kárate. Ella me dijo que no quería tener nadie en casa que supiera cómo matar gente (risas). Pero en 1965 tuve una operación de pecho. Cuando estaba dejando el hospital escayolado mi padre preguntó: “ialgún deseo?" Podía tener cualquier cosa que quisiera. Entonces, le dije que quería hacer kárate. Del hospital me llevo a una escuela de kárate. Entonces, el 15 de marzo, después de recuperarme, empecé en una escuela de kárate Shotokan.

\section{RT ¿Quién fue tu primer instructor? ¿Conseguiste tu cinturón negro con él?}

BR Peter Ventresca. Y si, conseguí con él mi cinturón negro, en 1972.

\section{RT Usted no se gana la vida como instructor de artes marciales. ¿A qué se dedica?}

BR Hago varias cosas diferentes. Un montón de cosas en la industria cinematográfica. Acrobacias y efectos especiales. Cuando me fui por primera vez a California, vivía en la Asociación de Jóvenes Cristianos. Oí un coche de bomberos y salí para ver lo que pasaba. Estaban filmando Towering Inferno [El coloso en llamas]. Conocí al encargado del vestuario de la película y en un momento dado me dijo, "simplemente pasea por allî". Si ves el video de El coloso en llamas, cerca del final de la película podrás verme paseando por detrás de los actores. Mucho más tarde estaba haciendo una demostración de artes marciales y un compañero vino y me preguntó que si podría coordinar el mismo tipo de cosas para efectos especiales en el teatro.

Si lees la introducción que escribí para el libro del sensei Kim, The classical Man (1992), verás lo que siento acerca de enseñar artes marciales como sustento. 


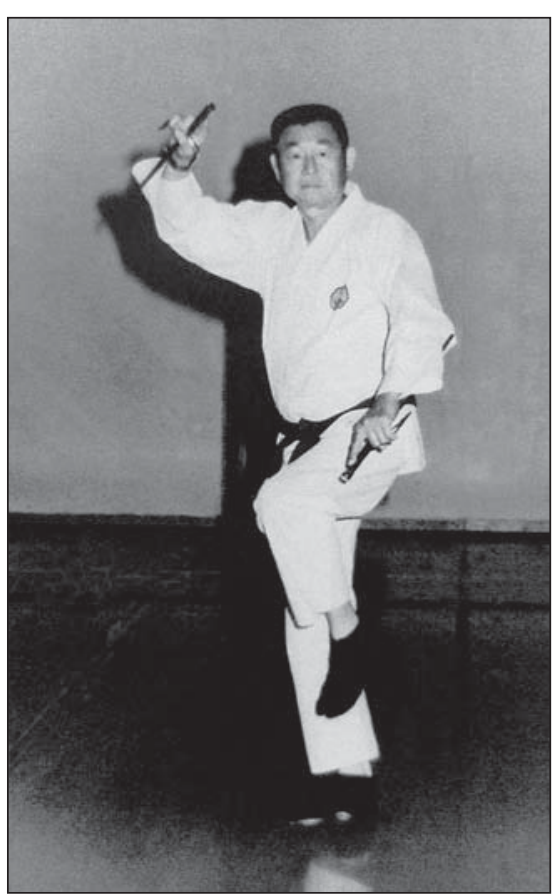

El Dr. Kim demostrando técnicas de sai.

\section{RT ¿Cuándo fue tu primer encuentro con el maestro Kim?}

BR Tenía 23 años y era julio de 1973. Había estado entrenando con Luis DeBacario unos dos años. Quería aprender con mayor profundidad, por eso le mandé una carta al sensei Kim. Me respondió que podía entrenar con él y así lo hice. Decidí dejar mi trabajo y conducir mi Buick de 1969 desde Boston a San Francisco. Llegué al dojo del maestro y me preguntó que cómo había llegado allí. Le dije que conduciendo. Me preguntó que si conducía. Dije, "si, mi coche está aparcado enfrente". Dijo "ya no”. Y cuando miré ¡habían remolcado mi coche (risas)! No conocía las normas de aparcamiento en la calle.

\section{RT ¿Entonces cómo era el entrenamiento con el maestro Kim?}

BR Había enérgicas repeticiones. Durante meses no hubo kata (formas), solo patadas, puñetazos, flexiones de tigre (sobre un brazo), estiramientos. Entonces me dijo que fuera al campamento de verano en San Diego. Enseñaba cinco o seis katas en una semana. En mis experiencias previas, aprendíamos puede que una kata por año. El maestro preguntó, “¿Cómo puedes aprender las katas más avanzadas sin conocer la kata básica?".

\section{RT ¿Qué enseñaba el maestro Kim en la Asociación de Jóvenes Cristianos Chinos?}

BR Taiji martes y jueves. Lunes, miércoles y sábados era combate, estilo libre con derribos, entonces allí hacíamos trabajo en colchoneta. Y el bastón (bo). Sencillamente entrenamiento y entrenamiento.

El maestro Kim siempre usaba las palabras "artes marciales". Decía que el kárate tenía límites. Tú tenías que llegar a ser un artista de la vida.

RT A lo largo de los años, debes haber conocido mucha gente que ha llegado y se ha ido. ¿Cómo cambió tu relación y tu posición con el maestro Kim?

BR Al principio solo le veía cuando cruzaba por el cuarto. Yo era invisible. Entonces en Francia, en 1975, me dijo, "ahora sé con quién voy a viajar". Y nunca fui excluido. Con el tiempo esto se transformó hasta hallarme como instructor auxiliar. Había depositado en mí su confianza y me preguntaba, “QQué debemos enseñar hoy?" Me sentía honrado de que tuviera en cuenta mi opinión. Al final era "Brian, dirige este evento".

Uno de sus cumplidos más grandes fue cuando me presentó a alguien en Sacramento. Dijo "Rose, te presento a mi amigo, Brian Ricci". Me impresionó que él me llamara su amigo.

\section{RT ¿Por qué el recibir el rango de dan del maestro Kim fue muy especial?}

BR Yo venía con las experiencias del Shotokan. Frank Gaviola ${ }^{5}$ tenía unos antecedentes distintos. Nos sentíamos algo así como artistas marciales completos. Pero tener el reconocimiento del maestro Kim fue algo difícil de ganar. El primer grado (shodan) y el segundo grado (nidan) fueron grados derivados del trabajo. Hubo muy pocos terceros grados (sandans). Otras organizaciones de fuera tenían sextos y séptimos grados, pero en ninguna parte eran ni de cerca tan buenos como eran los cinturones negros del sensei Kim. Fui cuarto grado durante muchos años. Las relaciones del cuarto dan fueron diferentes. Significaba que ya eras autosuficiente. Quería conocer todo sobre ti. El sensei Kim conocía tu personalidad. Conocía tu código moral en la vida.

Nunca le pedí un grado. Cuando el maestro Kim me dio el séptimo grado, nunca se lo dije a nadie. Él lo anunció en el banquete para celebrar su 80 cumpleaños y su décimo grado.

\section{RT ¿Cuál es el recuerdo más cariñoso del maestro Kim?}

BR El sentido del humor del sensei. Su risa. Nos encantaba verle moverse. Recuerdo la primera vez que en el campamento del verano hicimos una pequeña actuación mágica llamada "The Stubby Shapiro Show" [El espectáculo del rechoncho 
Shapiro]. Basado en un chiste que el maestro había contado. Pero, ese día, algo le había molestado. Realmente no estaba de buen humor. Pero gracias al espectáculo, el maestro Kim se rió tanto que tenía lágrimas en los ojos. Y me sentí muy bien de haber podido hacer algo así para cambiar su estado se ánimo.

\section{RT ¿Cuál es la cosa más importante que has aprendido del maestro Kim?}

BR Se feliz. Disfruta de la vida cada día. Vive el presente.

RT La mayoría de los estudiantes del sensei Kim están ahora entrenando contigo. ¿Qué legado del maestro Kim estás intentando inculcarles?

BR La tradición. El maestro Kim decía que si se conseguía fácil, no tenía valor. La tradición de las artes marciales ha sido conservada durante un largo tiempo.

\section{RT ¿Puede hablarnos sobre la International Zen Bei Butokukai?}

BR El maestro Kim eligió el nombre International Zen Bei Butokukai. Como Zen Bei significa "todo el mundo", refleja su extensión a todo el mundo. La forma del blasón que usamos muestra puramente nuestro respeto por Sensei Kim.

Después del fallecimiento del sensei Kim no dejó escritas instrucciones de cómo quería que continuaran las cosas. Sin embargo, me gusta pensar que ha sido lo mejor.

\section{RT ¿Cómo ves tu futuro en las artes marciales?}

BR Disfruto siendo un vehículo de paso de las enseñanzas del maestro Kim, desarrollando las habilidades de sus estudiantes de la mejor manera posible, y tratando a todos los estudiantes con honradez y continuando con su nivel de enseñanza.

\section{RT ¿Hay algo más que quiera decir?}

BR Todos hemos visto al maestro Kim como un individuo honorable. Yo he conseguido conocerle como hombre. Cometía errores. De hecho, su generosidad llegaba a ser un defecto. Siempre quería asegurarse de que sus estudiantes consiguieran lo mejor.

El Dr. Kim tenía el verdadero sentido de un sensei: "Uno que lo ha hecho antes" y compartía sus conocimientos con los estudiantes.

\section{Sección técnica}

El prototipo de la lanza (yari) fue traído originariamente del continente asiático a Japón y gozó de su mayor popularidad después de la invasión Mongola de finales del s. XIII (Draeger, 1973: 71, 72). Para los guerreros japoneses (samuráis), la lanza sólo era secundaria al arco y la flecha en la significación tradicional (Ratti y Westbrook, 1973: 241). Aún cuando los elaborados diseños de la punta de lanza sacaron a la misma fuera de la categoría de armas punzantes y le dieron nuevas funciones en acuchillamientos, enganches y desgarraduras, los mecanismos básicos del arte de la lanza continuaron sin cambiar, y los samuráis japoneses entrenaban ante todo para ser certeros con la estocada (Draeger, 1973: 72).

Es interesante anotar que el bastón (bo) fue desarrollado al mismo tiempo que el arte de la lucha con lanza y el límite entre las dos artes ha llegado a ser más bien impreciso. El bastón era comparativamente menos peligroso de practicar que la lanza y fue a menudo usado en las salas de entrenamiento de artes marciales (dojo) donde se enseñaba la lucha con lanza. En su tiempo, el uso de esta arma de madera llegó a estar tan bien desarrollado que los guerreros más cualificados podían involucrarse en un combate real usando el bastón (Ratti \& Westbrook, 1973:305, 308).

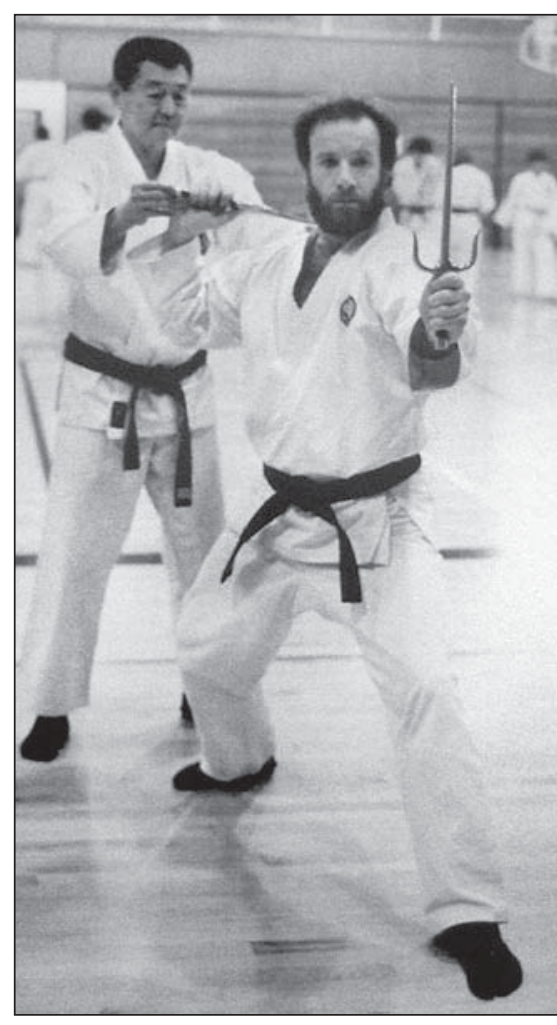

Brian Ricci ajustando su técnica de sai bajo la supervisión de su mentor. 
Aunque los japoneses habían usado armas de madera desde las primeras épocas, el bastón fue un arma humilde, porque hasta la persona más pobre podía tener uno. Debido a su efectividad, sin embargo, los samuráis no podían permitirse ignorar su estudio (Draeger, 1973: 76).

Yoshida Kotaro era conocido como un experto con la lanza (Corcoran, et al. 1993: 396) y Kim hablaba a menudo del entrenamiento con la lanza bajo su profesor (Kim, 1982: 35, 77, 82, 89). Ricci explicó que muchas de las katas practicadas con el bastón que se habían trasmitido gracias al sensei Kim, y que habían sido enseñadas en la Zen Bei Butokukai, eran realmente formas de lanza (Ricci, 2005, 21 de abril) La kata Yunigawa no kon fue pensada originariamente para ser realizada con la lanza (Ricci, 2005, 24 de mayo).

\section{Los movimientos de apertura de la Yunigawa no kon.}
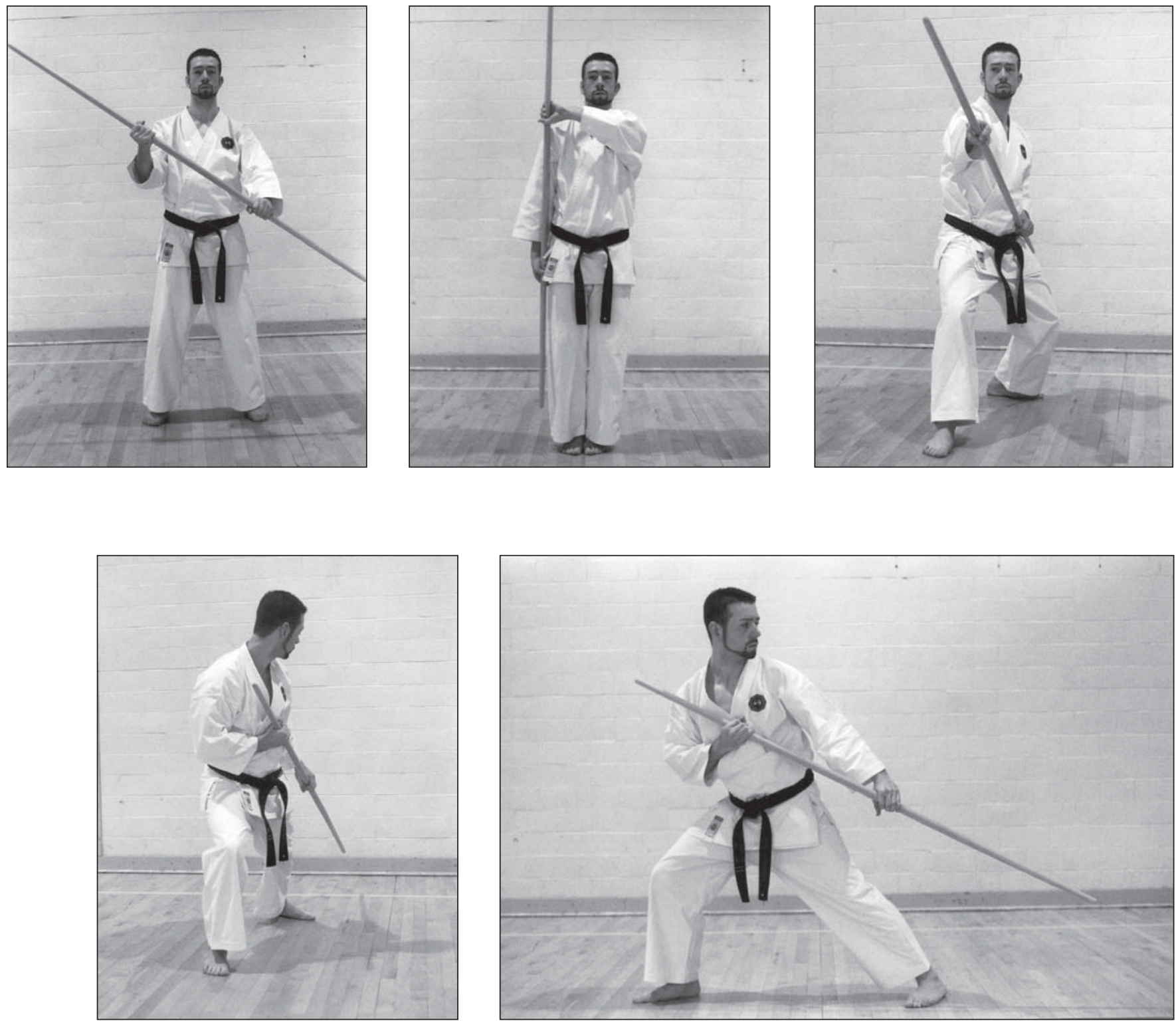

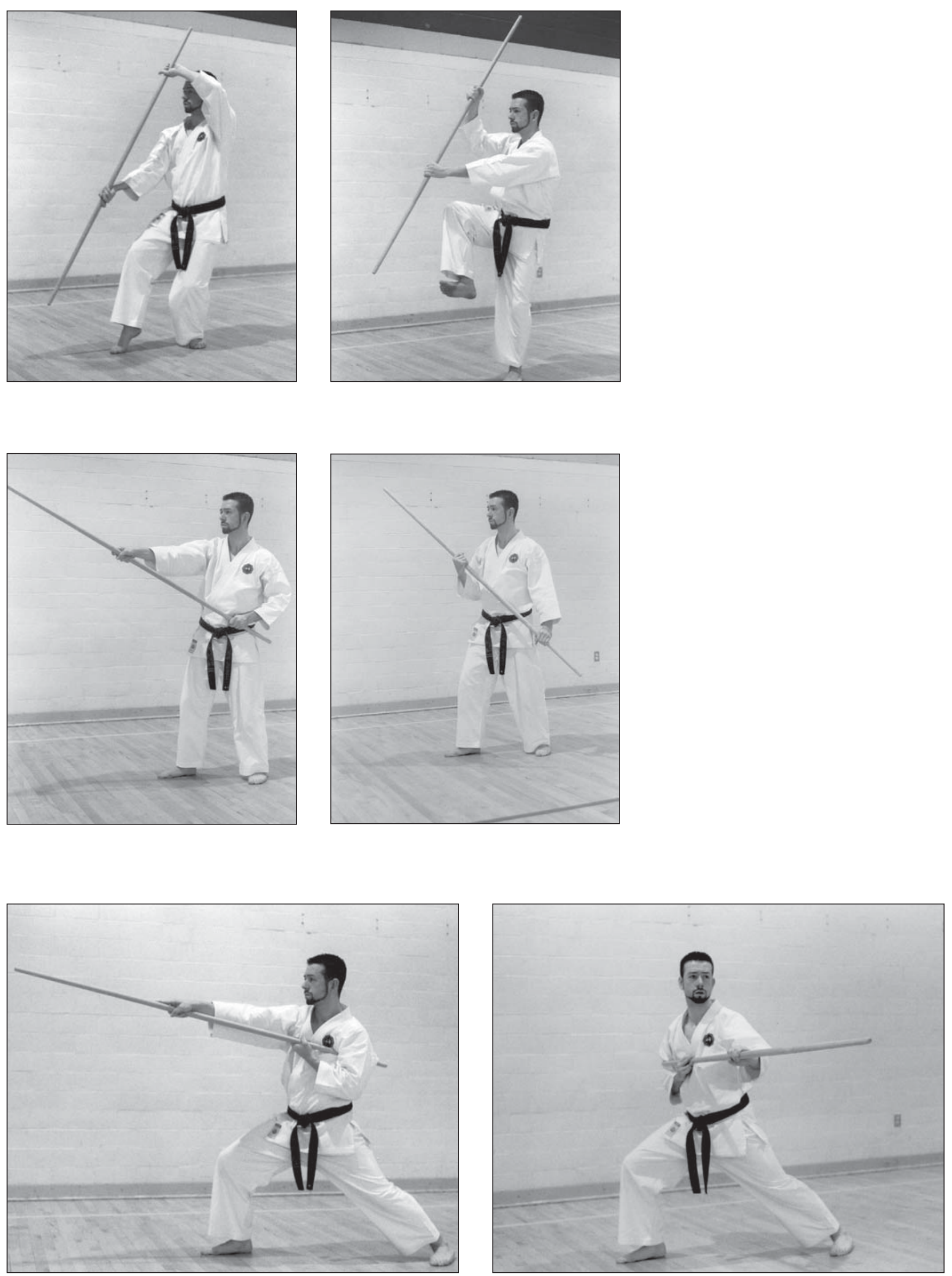

Revista de Artes Marciales Asiáticas 1 Volumen 1 Número 2 (62-75) - 2006 
Brian Ricci demuestra una combinación de la Yunigawa no kon. El segundo movimiento consiste en una retirada del bo para lanzar una estocada por debajo del bloqueo del oponente
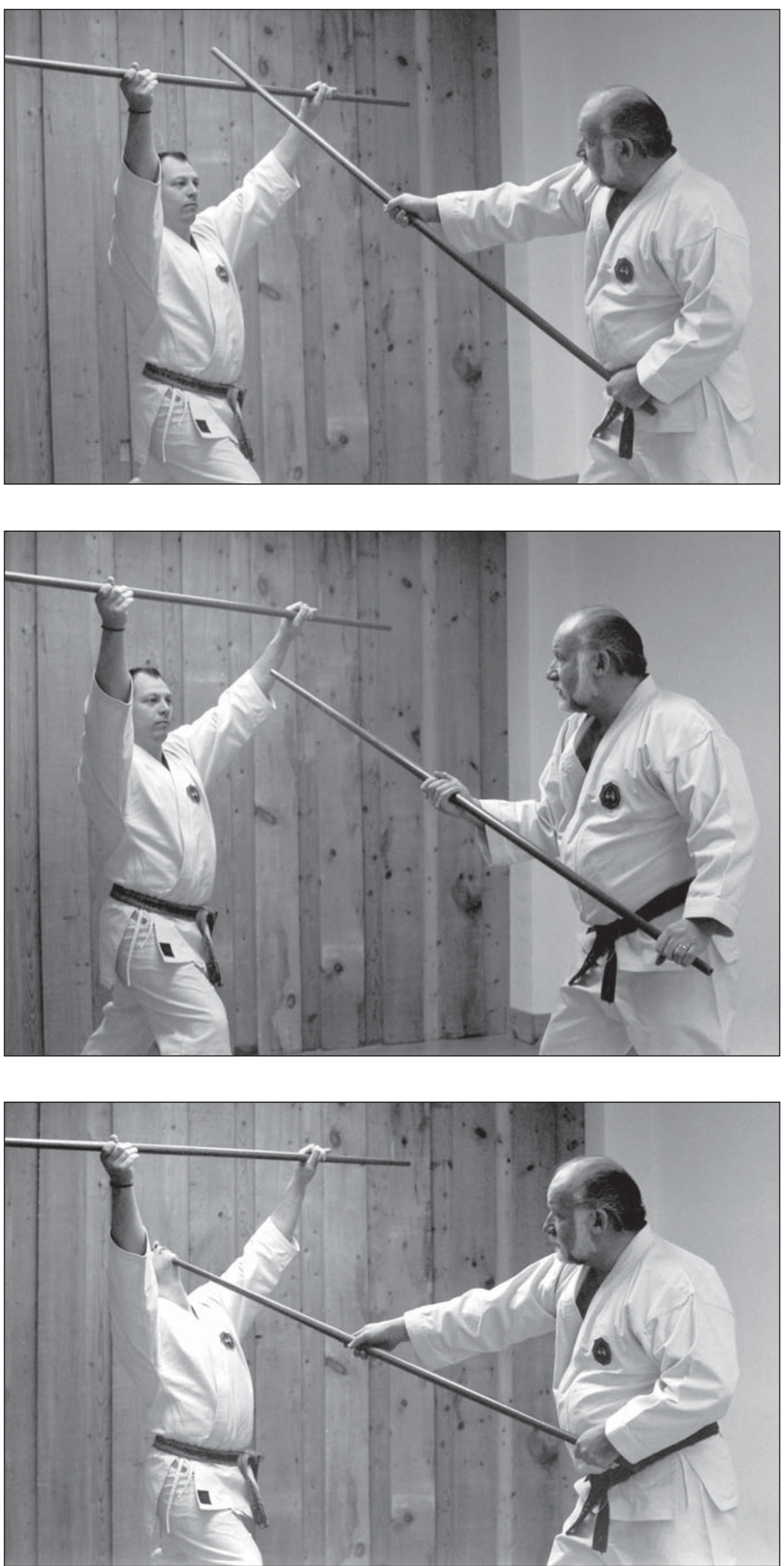


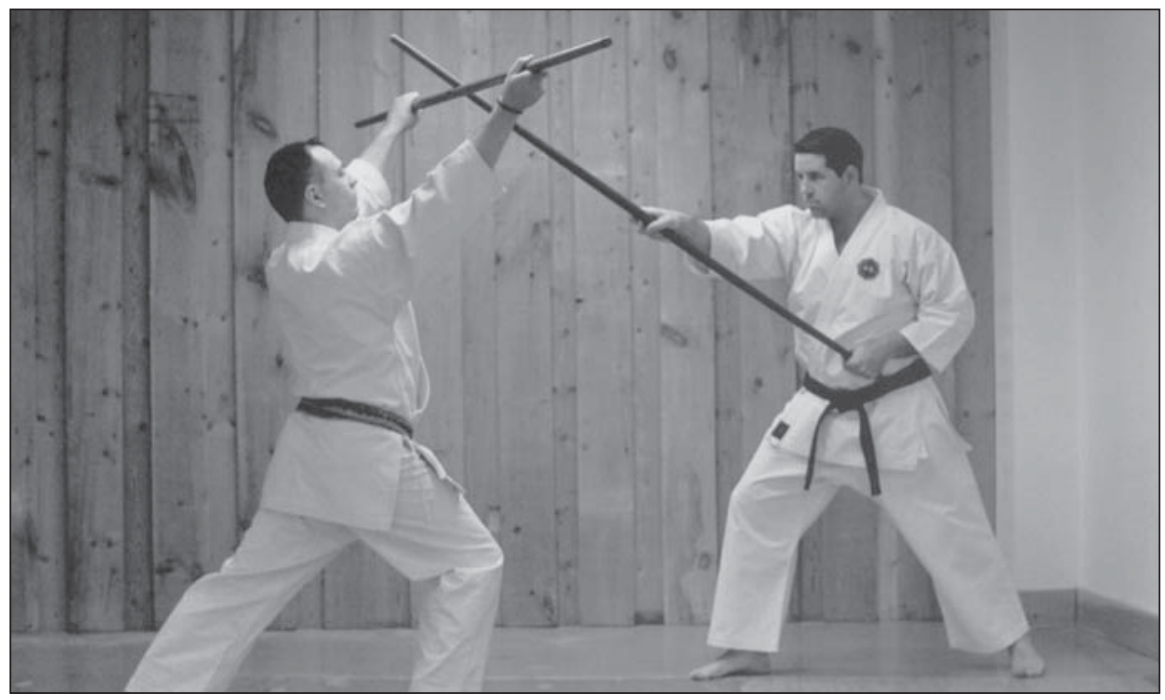

El bloqueo de yari o bo se

realiza con el bastón.

Estocada y retirada.
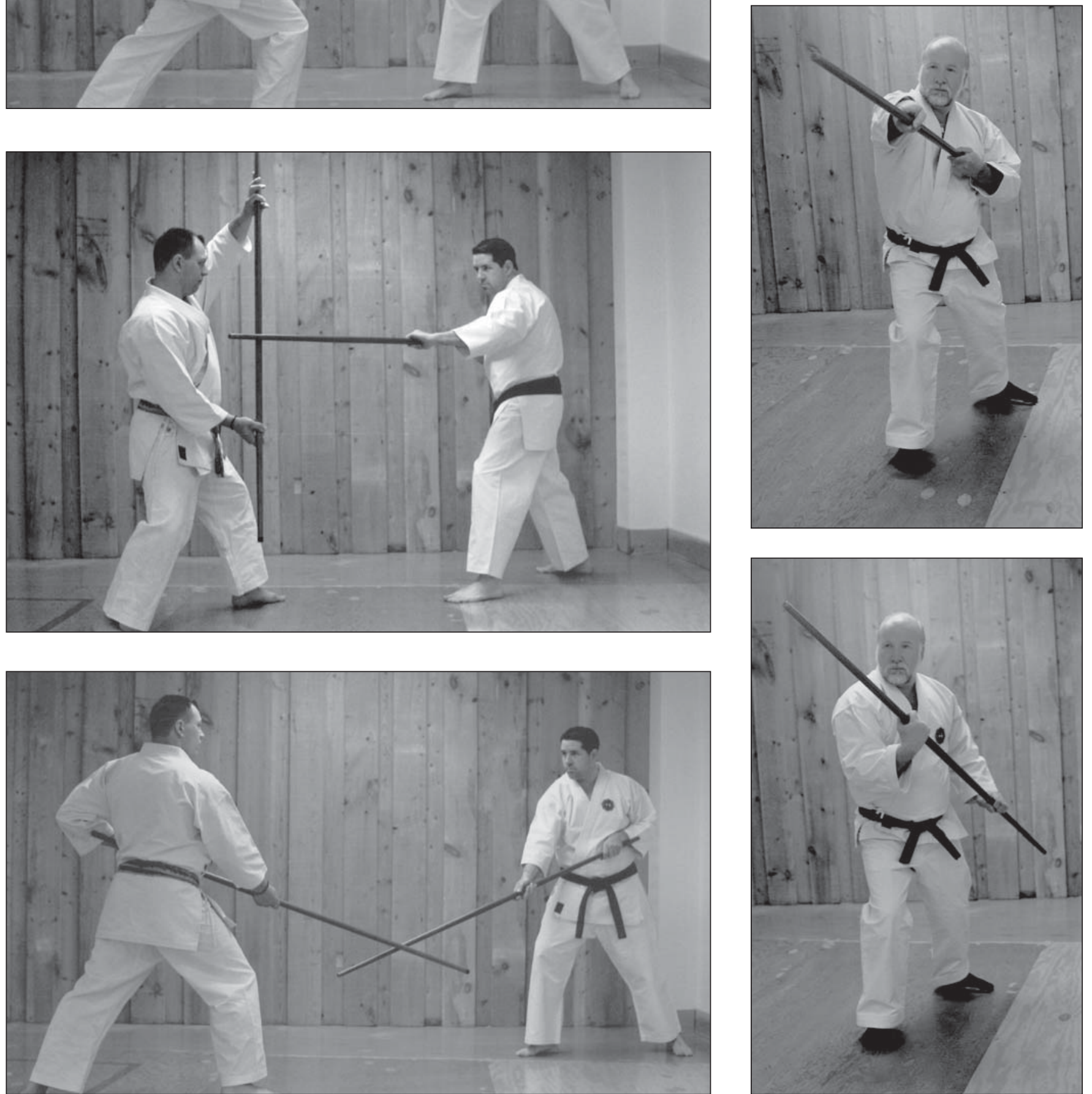

Revista de Artes Marciales Asiáticas 1 Volumen 1 Número 2 (62-75) - 2006 
Es importante utilizar un agarre apropiado con el yari/bo. Si la palma está orientada hacia arriba el arma puede soltarse con un golpe.
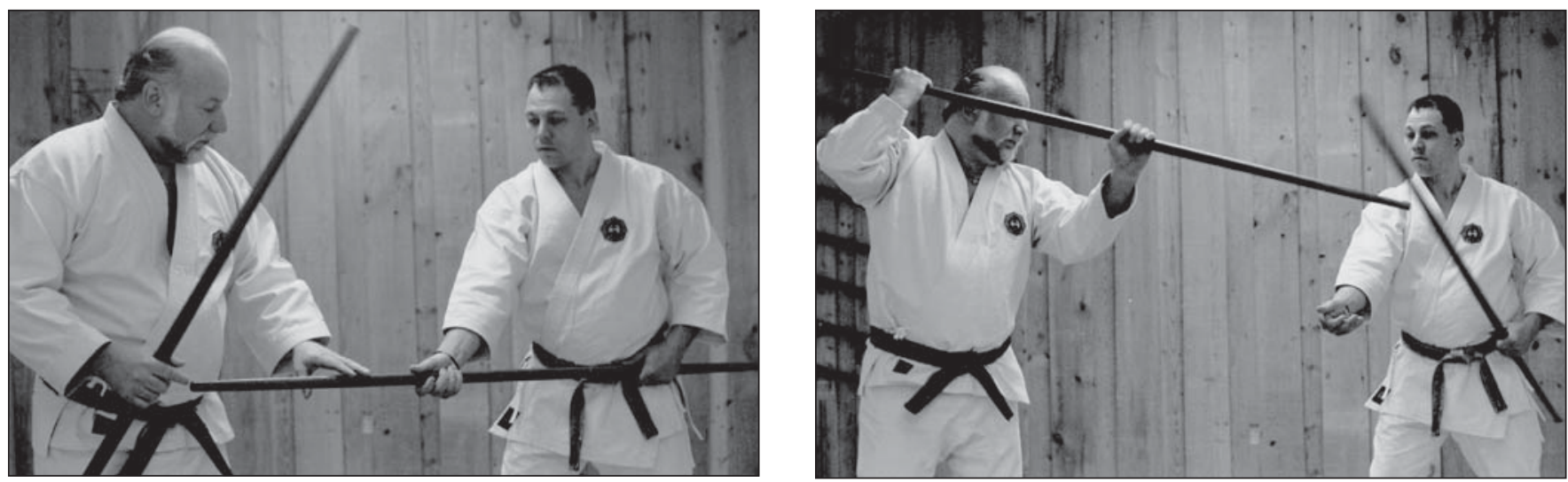

La palma tiene que mirar hacia abajo para controlar el arma.
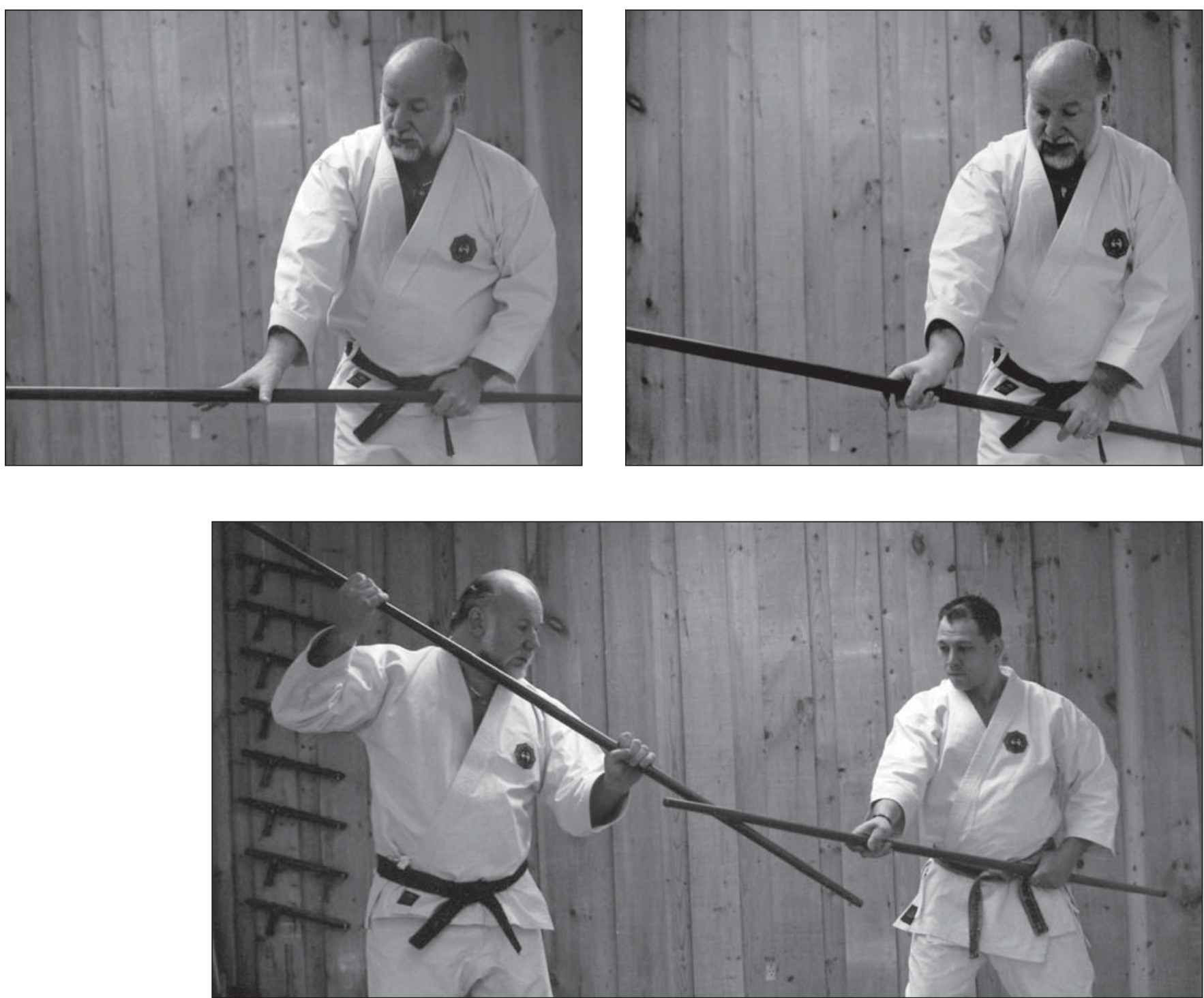
La estocada con el yari/bo se realiza con un movimiento

de torsión. Aquí Brian Ricci utiliza una técnica de

entrenamiento utilizada por su sensei, el Dr. Richard Kim.

El Dr. Kim hacía utilizar una toalla a sus estudiantes en las

estocadas para mostrar la torsión del yari/bo.
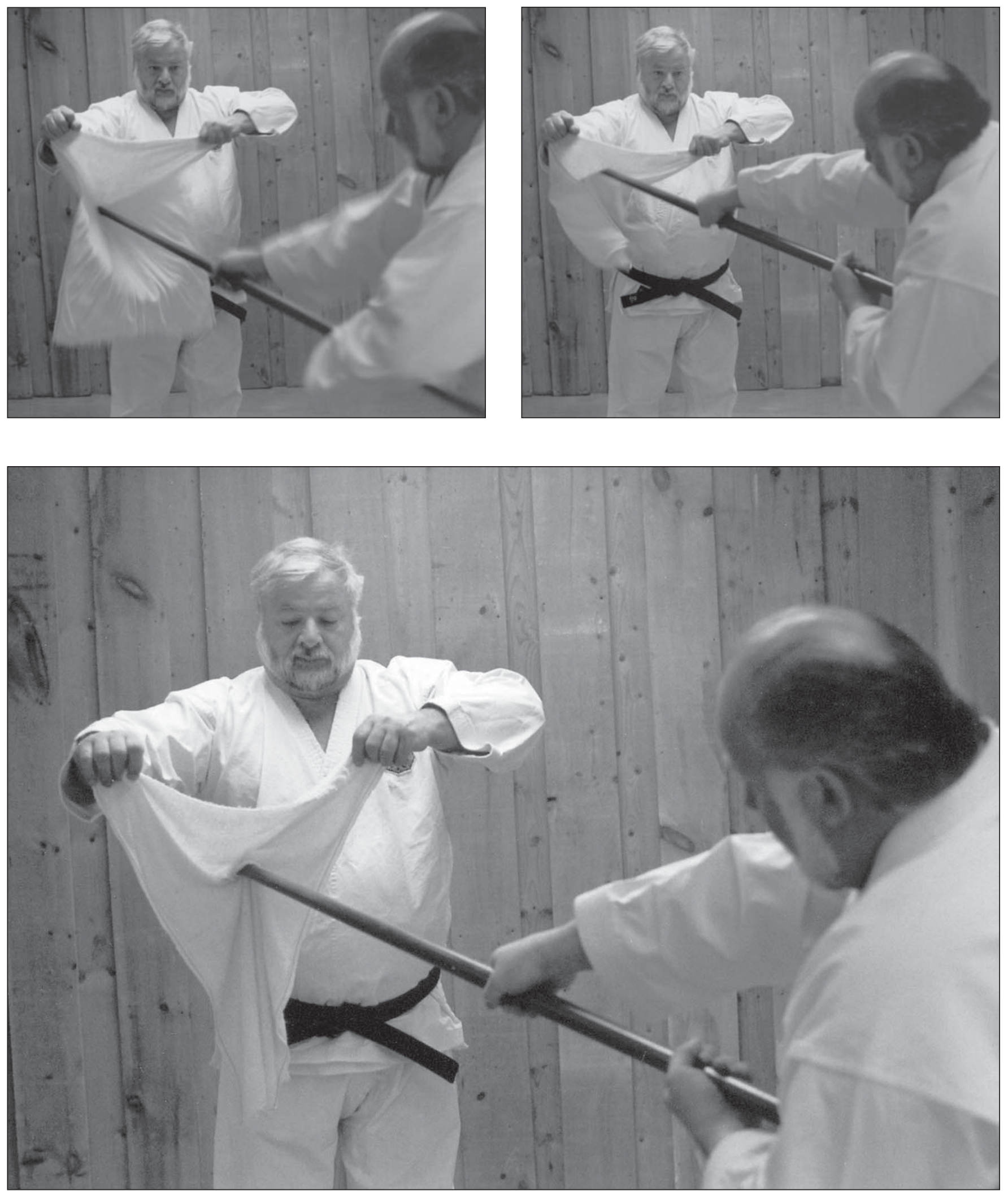


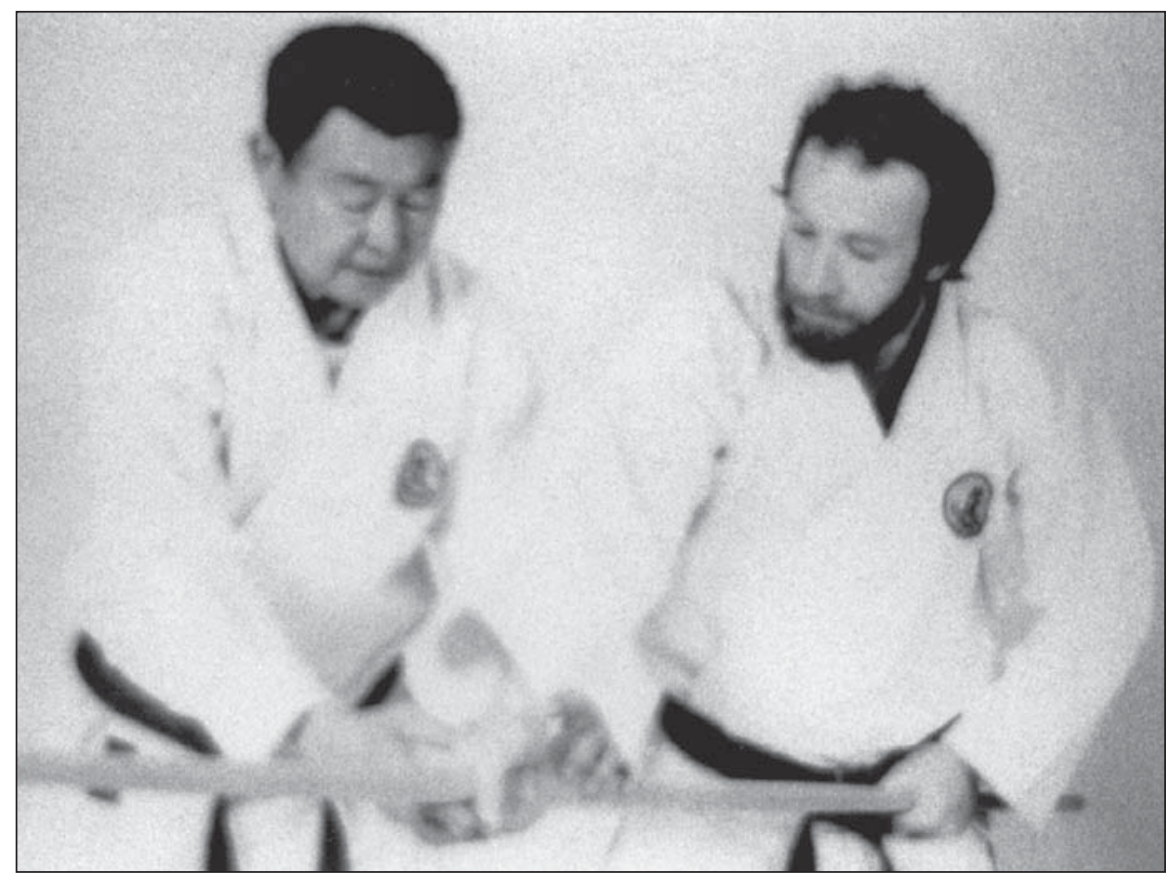

\section{Conclusión}

Nadie nunca podrá reemplazar al doctor Richard Kim. Las circunstancias de su vida le dieron la oportunidad de entrenar en numerosas artes marciales en sus países de procedencia durante tiempos turbulentos. Su desarrollo personal fue el resultado de su asombroso trabajo ético. El doctor Kim dijo una vez, "Si duermes más de cuatro horas al día, tu pierdes".

Hoy, tras cuatro años de su muerte, los estudiantes del Dr. Kim tienen enfrente la intimidante tarea de trasmitir las enseñanzas de su instructor. Su lealtad y devoción garantizan que el conocimiento y sabiduría del Dr. Kim puedan continuar para las siguientes generaciones. Y es seguro que esas generaciones verán las historias de la vida de este gran hombre como una leyenda.

\section{Notas}

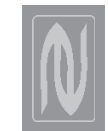

1 Oyama Masutatsu nació en Yee Hyung, en Kimje, Corea, en 1923. En 1937 fue enviado a una academia militar para chicos en Japón. Cambió su nombre y empezó a estudiar kárate Shotokan. Después de la guerra, Oyama estudió kárate Goju-ryu y con el tiempo creó su propio estilo, Kyokushinkai (Corcoran, et al., 1993: 365).

2 Kinjo Hiroshi nació el día de San Valentín de 1919. En un principio entrenó artes marciales con Hanashiro Chomo (1869-1945) y Oshiro Chojo (1888-1935) en Okinawa (McCarthy, 1994: 91).

3 Yamaguchi Gogen nació el 20 de enero de 1909. Cuando era niño entrenó esgrima japonesa (kendo) y kárate. En 1931 fue presentado al fundador del kárate Goju-ryu, Miyagi Chojun (Yamaguchi, s.f.: 75-77, 84).

4 Butokukai significa "Asociación de las Virtudes Marciales" (Draeger 1974: 35).

5 Frank Gaviola comenzó a entrenar con Richard Kim en 1968 en la Asociación de Jóvenes Cristianos en el Chinatown de San Francisco. Recibió su cinturón negro sexto grado en el 2001. Obtuvo su título de grado asociado en diseño arquitectónico del City College de San Francisco. Después de 25 años de carrera en la ingeniería, en 1992 dejó el trabajo para dedicarse a la enseñanza de las artes marciales a tiempo completo (Gould, 2002: 22). 


\section{Agradecimientos}

El autor quiere dar las gracias al sensei Brian Ricci por proporcionar algunas de las fotografías utilizadas en este artículo. Un agradecimiento especial para los maestros Ricci y Frank Gaviola por su ayuda y sus sugerencias con el artículo. También expreso un gran agradecimiento a Ed Ricci, John Wasilina, Dean y Tony Romanelli, y a Kelly Combs por aparecer en las fotografías. El autor quiere dar especialmente las gracias al Doctor Richard Kim por su instrucción.

\section{Bibliografía}

Castilonia, R. (1996). Nuggets in the ground. LaJolla, CA: International University Line.

Corcoran, J., Farkas, E. and Sobel, S. (1993). The original martial arts encyclopedia: Tradition-history-pioneers. Los Angeles: ProAction Publishing.

Draeger, D. (1973). Classical bujutsu. Tokyo: Weatherhill Inc.

Draeger, D. (1974). Modern bujutsu and budo. Tokyo: Weatherhill Inc.

Farkas, E. and Corcoran, J. (1983). The Overlook martial arts dictionary. New York: The Overlook Press.

Foley, S. (2005, 3 julio) Comunicación personal con el autor.

Gould, J. (ed.). (septiembre 2002). Sensei spotlight: Frank Gaviola. Martial Virtue, 22.

Kim, R. (1974). The weaponless warriors. Burbank, CA: Ohara Publications.

Kim, R. (1982). The classical man. Hamilton, Ontario: Masters Publication.

McCarthy, P. (1987). Classical kata of Okinawan karate. Santa Clarita, CA: Ohara Publications.

McCarthy, P. (1994). The world within karate \& Kinjo Hiroshi. The Journal of Asian Martial Arts, 3 (2): 90-99.

Ratti, O. and Westbrook, A. (1973). Secrets of the samurai. Rutland, VT: Charles E. Tuttle Company.

Ricci, B. (2005, 21 abril). Comunicación personal con el autor

Ricci, B. (2005, 4 mayo). Comunicación personal con el autor

Ricci, B. (2005, 17 mayo). Comunicación personal con el autor

Ricci, B. (2005, 22 mayo). Comunicación personal con el autor

Ricci, B. (2005, 24 mayo). Comunicación personal con el autor

Sells, J. (2000). Unante: The secrets of karate. Hollywood, CA: W.M. Hawley.

Svinth, J. (2001). Karate pioneer Yabu Kentsu, 1866-1937. Journal of Asian Martial Arts, $10(2): 8-17$.

Werrener, D. (s.f.). Memorial video-Sensei Richard Kim 1917-2001. Private production.

Warrener, D. (mayo 1982). Richard Kim: The weaponless warrior. Official Karate, 65.

Warrener, D. (junio 2001). Richard Kim: The classical man. Masters of Karate, 93.

Will, J. (s.f.). Gogen "the cat" Yamaguchi: The last interview. Fighter International, 24, 26.

Wingate, C. (1993). Exploring our roots: Historical and cultural foundations of the ideology of karate-do. Journal of Asian Martial Arts, 2 (3): 10-35.

Yamaguchi, G. (s.f.). Karate Goju-ryu by the cat. Tokyo: International Karate-do Goju-kai. 